\title{
ESTIMACIÓN DE LA BIOMASA DE COPA PARA ÁRBOLES EN PIE DE Pinus tropicalis MORELET EN LA EMPRESA FORESTAL INTEGRAL MACURIJE DE LA PROVINCIA DE PINAR DEL RÍO, CUBA
}

\author{
Alberto Vidal Corona ${ }^{1}$ \\ Jesús Rodríguez Rodríguez ${ }^{2}$ \\ Julio Yoan Benítez Naranjo ${ }^{3}$ \\ Roberto Carlos Álvarez Rivera ${ }^{4}$ \\ Humberto Gra Ríos
}

\begin{abstract}
RESUMEN
Los resultados obtenidos en este trabajo se presentan en forma de tablas elaboradas tomando como base los modelos matemáticos definidos a partir de las talas principales de bosques naturales de Pinus tropicalis Morelet mediante el empleo del análisis de regresión, para la cuantificación de la biomasa de copa (follaje y rama) de árboles en pie de la Empresa Forestal Integral Macurije, utilizando un muestreo completamente aleatorio por clases diamétricas. Se tomaron un total de 191 árboles que fueron talados, desramados y desfoliados, procediéndose al pesaje por separado, tanto de la biomasa foliar como de las ramas, de cada árbol, además de medir su diámetro a 1,3 metros del suelo y su longitud total.

Palabras clave: biomasa de copa, estimación, modelo matemático

\section{ESTIMATION OF CROWN BIOMASS FOR STANDING TREES OF Pinus tropicalis MORELET IN THE FOREST INTEGRATED ENTERPRISE MACURIJE IN THE PINAR DEL RÍO PROVINCE, CUBA.}

\begin{abstract}
Results offered in this paper are crowns weight tables (foliage and branches) contracted through equations derived from Pinus tropicalis Morelet natural forest using linear regression techniques. Data used were collected using a completely randomised design by diameter classes from 191 felled trees, cut the branches, strip off the leaves and weight them, one at a time. Besides, diameters at 1,3 meter above the ground level and total height were measured.

Key-words: crown biomass, estimation, mathematical model
\end{abstract}

\section{INTRODUCCION}

El nivel de desechos producidos, tanto en bosque como en las plantas procesadoras, constituye un obstáculo para la sustentabilidad de los bosques y las industrias forestales Noack (1995). En los últimos tiempos la necesidad de la estimación racional e integral de toda la biomasa forestal aprovechable, así como la predicción de los incrementos potenciales de la misma, se han convertido en un importante aspecto a tener en cuenta dada la enorme cantidad de alternativas de su uso, tanto dentro de la planificación productiva como de sus posibilidades de desarrollo científico técnico (Ritchie y Hann, 1990).

La biomasa renovable cultivada es un combustible neutro en cuanto a la emisión de dióxido de carbono con un bajo contenido sulfúrico que puede convertirse en electricidad, calor, y combustibles líquidos o gaseosos. La biomasa se cultiva permanentemente para

\footnotetext{
${ }^{1}$ Ing. Forestal, Dr. C. Investigador Auxiliar, e-mail: iif@ip.etecsa.cu

${ }^{2}$ Ing. Forestal, M. Sc. Investigador Auxiliar, e-mail: eefv@ vega.inf.cu

${ }^{3}$ Ing. Forestal, M. Sc. Investigador Aspirante Estación Experimental Forestal Camagüey e-mail: estafor@minag.cmw.inf.cu

${ }^{4}$ - Ing. Forestal Estación Experimental Forestal Viñales.

5 - Ing. Forestal Investigador Titular Instituto de Investigaciones Forestales
}

Recebido para publicação: 20/02/2002

Aceito para publicação: 28/06/2002 
generar energía a fin de producir beneficios ambientales, como la conservación de los suelos y la protección de la biodiversidad en comparación con los cultivos anuales. Además las comunidades rurales pueden obtener empleo en lugar de excluir los suelos del proceso productivo con el único fin de secuestrar el carbono. Por lo tanto el cultivo y la producción de biomasa para energía tiene numerosas ventajas sociales y ecológicas (Hall, 1998).

La biomasa forestal se ha convertido en un importante elemento en los estudios sobre los cambios que ocurren a escala mundial. A partir de la biomasa forestal se puede calcular la concentración de carbono en la vegetación (aproximadamente el 50\% de la biomasa está formada por carbono) y por consiguiente se pueden hacer estimaciones sobre la cantidad de dióxido de carbono que entra a la atmósfera cada vez que se desmonta o se quema un bosque. La biomasa es una variable que sirve también para comparar las características estructurales y funcionales de un ecosistema forestal en un amplio abanico de condiciones (FAO, 1995).

Este trabajo tiene como objetivo obtener ecuaciones matemáticas que permitan elaborar tablas de estimación de la biomasa de copa foliar y de ramas de Pinus tropicalis Morelet en áreas de la empresa Macurije.

\section{MATERIALES Y METODOS}

Se trabajó con la información procedente de los bosques naturales de Pinus tropicalis Morelet de la Empresa Forestal Integral Macurije, donde se talaron un total de 191 árboles empleando un muestreo completamente aleatorio por clases diamétricas. A cada uno de ellos se le midió el diámetro a 1,3 metros sobre el nivel del suelo $\left(\mathrm{d}_{1,3}\right)$ y la altura total $(\mathrm{h})$, siendo posteriormente desramado y desfoliado, realizándose en cada caso el pesaje, por separado, de toda la biomasa verde, tanto del follaje (P. F.), como de las ramas (P. R.).

La ecuación general empleada para el cálculo fue la siguiente:

$\log$ Peso $(F)(R)=a+b \times \log \left(d_{1,3}\right)+C$

Donde:

Log: Logaritmo de base 10.

Log Peso (F): Logaritmo del peso del follaje
Log Peso (R): Logaritmo del peso de ramas a y b: Constantes.

$\left(\mathrm{d}_{1,3}\right)$ : Diámetro del árbol a 1,3 metros sobre el nivel del suelo.

C: Factor de Corrección

Toda la información obtenida se agrupó por clases diamétricas, trabajándose para estimar las variables dependientes, peso foliar y peso de las ramas de copa, a partir de dos variables independientes (diámetro normal y altura); empleando con este objetivo las transformaciones cuadráticas y logarítmicas de las variables.

En la construcción de los modelos matemáticos se emplearon las variables independientes de mayor correlación lineal con las variables dependientes estudiadas, en función de la matriz de correlación efectuada, sometiéndose las mismas al análisis de regresión "paso a paso", seleccionándose las ecuaciones de mejor ajuste y atendiendo al menor número de variables y mayor facilidad de manejo práctico.

Para obtener el peso seco de la biomasa de copa foliar y de ramas se emplearon los valores constantes 0,58 y 0,71 respectivamente, obtenidos para cada caso a partir de la relación existente entre el peso verde y el peso seco (PV/PS).

Para definir el grado de confiabilidad de las tablas obtenidas se determinó la Desviación Global (\%), teniendo en cuenta las distintas clases diamétricas y las alturas de los árboles, comparando en cada caso el valor real en peso $(\mathrm{kg})$ de acículas y de las ramas con el valor teórico de las tablas.

Para validar las ecuaciones empleadas en la estimación del peso de la biomasa de copa foliar y maderable se realizó el cálculo de la Desviación global con la información procedente de 188 árboles totalmente ajenos a los utilizados en la tabla confeccionada, mediante la siguiente ecuación:

D.G $=\sum$ P.R $-\sum$ P.C $/ \sum$ P.C $* 100$

Donde:

D.G: Desviación Global

P.R : Peso Real $(\mathrm{kg})$

P.C : Peso Calculado (kg) 
La calidad del ajuste se valoró mediante los indicadores clásicos del análisis de regresión Cailliez (1980), coeficiente de correlación (R), coeficiente de determinación (R2) y desviación standard de la estimación (S), efectuándose a continuación el análisis de los residuales de los modelos de mejor ajuste obtenidos.

\section{RESULTADOS Y DISCUSIÓN}

Como resultado del proceso de la información disponible se confeccionaron las tablas de peso de follaje y peso de las ramas de Pinus tropicalis Morelet, teniendo como fundamento de cálculo un modelo matemático en cada caso, definidos a partir de la biomasa que queda abandonada en el bosque (Coutinho et al, 1987; Rivas et al, 1990).

Tabla 1: Modelos empleados para estimar el peso de la biomasa de copa

\begin{tabular}{|c|c|c|c|c|c|}
\hline Modelos & $\mathbf{R}$ & $\mathbf{R}^{\mathbf{2}}$ & $\mathbf{S}$ & DG & Factor C \\
\hline Log P. F. $=-1,4022+2,0357 * \log d+$ C & 0,9789 & 0,9583 & 0,1404 & $-0,46$ & 0,2791041 \\
\hline Log P. M. $=-1,9292+2,6177 * \log d+$ C & 0,9799 & 0,9602 & 0,1763 & 1,5 & 0,4406161 \\
\hline
\end{tabular}

Del análisis de regresión efectuado se obtuvieron, para el peso de biomasa foliar y peso de las ramas de copa verde, ecuaciones logarítmicas con la variable independiente d1,3 (tabla 1), siendo necesario destacar que en todos los casos estudiados el peso foliar y el peso de las ramas aumenta de forma directamente proporcional al diámetro normal de los árboles (Kill, 1980; Malleux, 1982; Wade, 1989 y Payanden, 1991). (Ver gráfico 1)

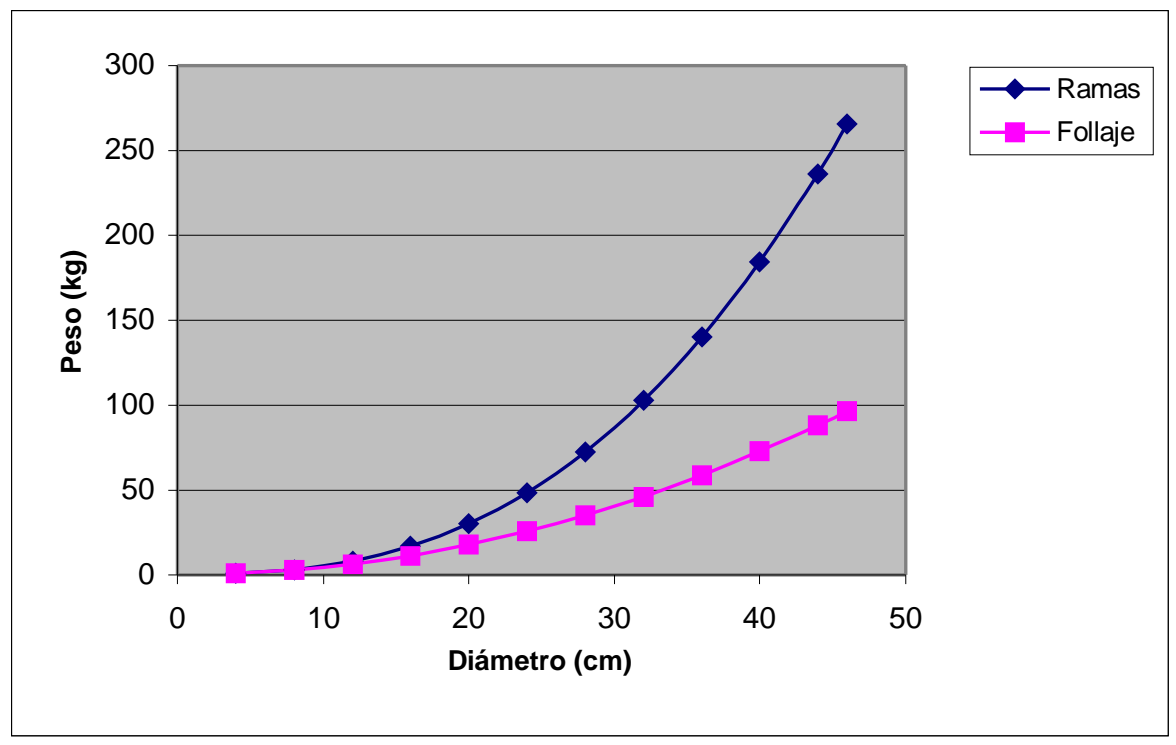

Gráfico 1: Comportamiento de la biomasa de copa foliar y de ramas por clase diamétrica

Como se puede apreciar las ecuaciones seleccionadas para realizar la estimación solo incluyen el diámetro normal de los árboles como variable independiente. Esto sucede porque a pesar de que la altura está relacionada con las variables dependientes estimadas su aporte a los modelos no fue significativo construyéndose por esta razón tablas de una sola entrada.

En función con el análisis de regresión llevado a cabo, todos los modelos obtenidos mostraron altos coeficientes de determinación $(\mathrm{R} 2 \geq 0,9)$. Además, por el análisis efectuado a los residuales se pudo comprobar el cumplimiento de los supuestos teóricos del análisis de regresión (Gujarati, 1999; Hair et al, 1999).

Para un uso simple del principio de transformación, en asociación con los cálculos realizados, se utiliza el factor de corrección para el sesgo resultante de las transformaciones a las variables dependientes de los modelos 
seleccionados para realizar la estimación de la biomasa de copa. Este asume que los errores son homogéneos y están normalmente distribuidos. El factor de corrección se debe a Meyer (1944) citado por Alder (1980).

Los resultados obtenidos con la determinación de la Desviación Global muestran una buena calidad de los modelos definidos (D.G. $= \pm 10 \%$ ), acorde con las exigencias de la literatura internacional. Los valores calculados a partir de las ecuaciones seleccionadas para estimar la biomasa de copa foliar y de ramas, determinados por clase diamétrica, se observan en la tabla 2.

Tabla 2: Peso estimado de biomasa foliar y maderable de copa de Pinus tropicalis Morelet por clase diamétrica. $(\mathrm{kg})$

\begin{tabular}{|c|c|c|c|c|}
\hline CD (cm) & Follaje Verde & Follaje seco & Ramas Verdes & Ramas Secas \\
\hline 4 & 0,95 & 0,55 & 0,88 & 0,63 \\
\hline 5 & 1,33 & 0,77 & 1,24 & 0,88 \\
\hline 6 & 1,80 & 1,04 & 1,72 & 1,22 \\
\hline 7 & 2,36 & 1,37 & 2,36 & 1,68 \\
\hline 8 & 3,01 & 1,75 & 3,16 & 2,25 \\
\hline 9 & 3,75 & 2,17 & 4,14 & 2,94 \\
\hline 10 & 4,58 & 2,66 & 5,32 & 3,78 \\
\hline 11 & 5,50 & 3,19 & 6,70 & 4,76 \\
\hline 12 & 6,51 & 3,78 & 8,31 & 5,90 \\
\hline 13 & 7,62 & 4,42 & 10,14 & 7,20 \\
\hline 14 & 8,81 & 5,11 & 12,22 & 8,67 \\
\hline 15 & 10,10 & 5,86 & 14,55 & 10,33 \\
\hline 16 & 11,47 & 6,66 & 17,14 & 12,17 \\
\hline 17 & 12,94 & 7,51 & 20,02 & 14,21 \\
\hline 18 & 14,51 & 8,41 & 23,18 & 16,46 \\
\hline 19 & 16,16 & 9,37 & 26,63 & 18,91 \\
\hline 20 & 17,91 & 10,39 & 30,40 & 21,58 \\
\hline 21 & 19,75 & 11,46 & 34,48 & 24,48 \\
\hline 22 & 21,69 & 12,58 & 38,89 & 27,61 \\
\hline 23 & 23,71 & 13,75 & 43,63 & 30,98 \\
\hline 24 & 25,84 & 14,98 & 48,72 & 34,59 \\
\hline 25 & 28,05 & 16,27 & 54,17 & 38,47 \\
\hline 26 & 30,36 & 17,61 & 59,98 & 42,58 \\
\hline 27 & 32,76 & 19,00 & 66,16 & 46,97 \\
\hline 28 & 35,26 & 20,45 & 72,72 & 51,63 \\
\hline 29 & 37,85 & 21,95 & 79,68 & 56,57 \\
\hline 30 & 40,53 & 23,51 & 87,03 & 61,79 \\
\hline 31 & 43,31 & 25,12 & 94,79 & 67,30 \\
\hline 32 & 46,18 & 26,79 & 102,97 & 73,11 \\
\hline 33 & 49,15 & 28,51 & 111,57 & 79,21 \\
\hline 34 & 52,21 & 30,28 & 120,60 & 85,62 \\
\hline 35 & 55,37 & 32,11 & 130,07 & 92,35 \\
\hline 36 & 58,62 & 34,00 & 139,99 & 99,39 \\
\hline 37 & 61,97 & 35,94 & 150,37 & 106,76 \\
\hline 38 & 65,41 & 37,94 & 161,21 & 114,46 \\
\hline 39 & 68,94 & 39,99 & 172,52 & 122,49 \\
\hline 40 & 72,97 & 42,09 & 184,31 & 130,85 \\
\hline 41 & 76,30 & 44,26 & 196,59 & 139,58 \\
\hline 42 & 80,12 & 46,47 & 209,36 & 148,65 \\
\hline 43 & 84,04 & 48,74 & 222,63 & 158,07 \\
\hline 44 & 88,06 & 51,07 & 236,42 & 167,86 \\
\hline 45 & 92,16 & 53,46 & 250,71 & 178,01 \\
\hline 46 & 96,37 & 55,89 & 265,54 & 188,53 \\
\hline
\end{tabular}




\section{CONCLUSIONES}

La variable independiente más eficiente para la estimación en pie del peso de la biomasa de copa (foliar y ramas) de la especie estudiada fue el diámetro a 1,3 metros sobre el nivel del suelo (d1,3), por lo que se considera que para la cuantificación de dicha biomasa, en bosques naturales de Pinus tropicalis Morelet de la Empresa Forestal Integral Macurije, pueden ser utilizados los modelos matemáticos definidos en este trabajo, así como las tablas elaboradas a partir de los mismos.

\section{REFERENCÍAS}

ALDER, D. (1980) Estimación del volumen forestal y predicción del rendimiento con referencia especial a los trópicos. Estudios FAO Montes 22/2. Roma, Italia. 118 p.

CAILLIEZ, F. (1980) Estimación del volumen forestal y predicción del rendimiento. Vol. 1. Estimación del volumen. Estudios FAO Montes. 22/1. Roma, Italia. 92 p.

CRUZ; E. C. da \& MACHADO, S. A. (1987) Seleção de equacões para a estimativa de resíduos florestais para fins energéticos numa floresta tropicãl. Floresta. Revista do Centro de Pesquisas Forestais. Vol. XVII. No. 1 e 2.

FAO (1995) Evaluación de los recursos forestales 1990, Países Tropicales. Estudio FAO Montes. Roma, Italia. p $32-36$

GUJARATI, D. N. (1999) Econometría Segunda parte. Estados Unidos. p 120 - 131

HAIR, J. F.; R. E. Anderson; R. L. Tatham; W. C. Blanck (1999) Análisis multivariante, Quinta edición. Prentice Hall Iberia. Madrid, España. 832 p.
HALL, D. (1998) Sustitución de combustibles fósiles a sumideros de carbono. Las repercusiones del uso de la biomasa para el protocolo de Kyoto. Actualidad Forestal Tropical. Vol. 6, $\mathrm{N}^{\circ}$ 4. OIMT. p9 - 10

KILL, A. D (1980) Fuel weight tables for White Spruce and Lhodgepole Pine in Alberta. Ottawa. Minister of Forestry and Rural Development. $13 \mathrm{p}$.

MALLEUX, J. (1982) Inventarios forestales en bosques tropicales. Lima, U.N.A. 414 p.

NOACK, D. (1995) Cómo aprovechar mejor las maderas de los trópicos. Actualidad Forestal Tropical. Vol. 3, No 2. OIMT. p 12 $-13$

PAYANDEN, B. (1991) Choosing regression models for biomass prediction equations. Forestry Chronicle. 57 (5): 229-232.

RITCHIE, M. W.; D. W. Hann (1990) Equations for predicting the 5-year height of six conifer species in Southwest Oregon. Oregon State University. Research Paper. 54. USA. 12 p.

RIVAS, L.; A. Catalán; E. Arenda (1990) Biomasa y contenido de nutrientes del Brosimun alicastrum y Pouteria anibaefolia en la reserva forestal de Caparo, Caparo. Estado de Barinas. Revista Forestal Venezolana. XXIV: (34): 29-44.

WADE, D. (1980) Estimating slash quantity from standing loblolly pine. U.S. Forest Service Res. Int- 197. Note SE-125. 156 p. 